九州大学学術情報リポジトリ

Kyushu University Institutional Repository

\title{
TENEBRIONIDAE OF THE NANSEI ISLANDS IV (COLEOPTERA)
}

Chujo, Michitaka

https://doi.org/10.5109/2378

出版情報 : ESAKIA. 12, pp.1-16, 1978-11-30. Entomological Laboratory, Faculty of Agriculture， Kyushu University

バージョン :

権利関係 : 
ESAKIA, (12) : 1-16. 1978

\title{
TENEBRIONIDAE OF THE NANSEI ISLANDS IV (COLEOPTERA)*
}

\author{
Michitaka ChûJô \\ Hikosan Biological Laboratory, Faculty of Agriculture \\ Kyushu University, Hikosan. Fukuoka 824-07, Japan
}

\begin{abstract}
Forty-nine species of the Tenebrionidae are recorded from Ishigakijima Island, 27 of which are new to the fauna of the island. Three new taxa are described, viz.: Platydema planiconnis sp. nov. from this island, Hemicera sakishimensis sp. nov. from this island, Miyakojima Island, Iriomotejima Island and Yonakunijima Island, and Phaedis (Phaedis) marmoratus sp. nov. from this island and Iriomotejima Island.
\end{abstract}

\section{ISHIGAKI JIMA I.}

\section{PEDININI}

†1. Diphyrrhynchus iriomotensis M. T. Chûjô, 1966

Spec, exam.: 3 exs., Yoshihara-Arakawa, Ishigaki, Japan, 17. iv. 1974, Coll. H. Irie.

\section{O PATRINI}

2. Gonocephalum coenosum Kaszab, 1952

Spec. exam.: 4 exs., Is. Ishigaki, Loo-Choo, 1, ii. 1957, Coll. M. Nagayama; 25 exs., Hoshino, Ishigaki Is., Ryukyu, 6. i. 1961, K. Nozato leg. ; 1 ex., Ishigaki I., Ryukyus, 3. iv. 1969, R. Takubo leg, ; 1 ex., Ishigaki-shi, Is. Ishigaki, 3. iv. 1969, Coll. M. Chûjố; 1 ex., Mt. Banna, Ishi., Ryukyu Is., 11. iv. 1969, H. Makihara leg. ; 1 ex., Mt. Banna-dake, Is. Ishigaki, 28. viii. 1970, Coll. M. Chûjô; 1 ex., Ishigaki Air Port, Ishigaki, 28. viii. 1970, Coll. M. Chûjô; 4 exs., Botanical Garden, Banna, Ishigaki I., Ryukyus, 12. vi. 1974, H. Makihara leg.; 2 exs. (at light), Mt. Banna, Ishigaki I., Ryukyus, 14. vi. 1974, H. Makihara leg.; 1 ex., Botanical Garden, Banna, Ishigaki I., Ryukyus, 15. vi. 1974, H. Makihara leg.; 2 exs., Arakawa, Ishigaki I., Ryukyus, 18. vi. 1974, H. Makihara leg.; 1 ex.,

\footnotetext{
* Contribution from the Hikosan Biological Laboratory, Faculty of Agriculture, Kyushu University, Hikosan (Ser. '2, No. 38).

$\dagger$ The dagger shows that the species has hitherto been unrecorded from the island.
} 
Arakawa, Ishigaki I., Ryukyus, 26. vi. 1974, H. Makihara leg. ; 7 exs., Yoshihara, Ishigaki I., Ryukyus, 28. v. 1975, H. Makihara leg.

TRACHYSCELINI

†3. Trachyscelis chinensis Champion, 1894

Spec. exam. : 4 exs., Yoshihara-Arakawa, Ishigaki, Japan, 17. v. 1974, Coll. H. Irie.

\section{PHALERIINI}

$\nmid$ 4. Micropedinus pallidipennis Lewis, 1894

Spec. exam. : 1 ex., Yoshihara-Arakawa. Ishigaki, Japan, 17. v. 1974, Coll. H. Irie.

\section{BOLITOPHAGINI}

5. Dicraeosis carinatus carinatus Gebien, 1913

Spec. exam.: 2 exs., Mt. Banna, Ishi., Ryukyu Is., 24. viii. 1969, H. Makihara leg. ; 2 exs., Mt. Banna, Ishi., Ryukyu Is., 7-9. ix. 1969, H. Makihara leg.; 1 ex., Mt. Omoto, Ishigaki I., Ryukyus, 11. vii. 1970, H. Makihara leg. ; 2 exs., Hoshino, Ishigakijima, Nansei Is., Japan, 19. iv. 1973, Coll. H. Irie ; 1 ex., Mt. Banna, Ishigaki I., Ryukyus, 9. iv. 1974, K. Kusama leg. ; 3 exs., Arakawa, Ishigaki I., Ryukyus, 7. vi. 1974, н. Makihara leg. ; 5 exs., Botanical Garden, Banna Ishigaki I., Ryukyus, 10. vi. 1974, H. Makihara leg.; 1 ex., Botanical Garden, Banna, Ishigaki I., Ryukyus, 12. vi. 1974, H. Makihara leg.; 8 exs., Botanical Garden, Banna, Ishigaki I., Ryukyus, 14. vi. 1974, H. Makihara leg. ; 5 exs., Arakawa, Ishigaki I., Ryukyus, 18. vi. 1974, H. Makihara leg. ; 3 exs., Mt. Omoto, Ishigaki I., Ryukyus, 20. vi. 1974, H. Makihara leg.; 2 exs., Mt. Banna, Ishigaki I., Ryukyus, 25. vi. 1974, H. Makihara leg.; 1 ex., Arakawa, Ishigaki I., Ryukyus, 26. vi. 1974, H. Makihara leg.; 1 ex.(at light), Mt. Banna, Ishigaki I., Ryukyus, 26. vi. 1974, H. Makihara leg.; 1 ex., Mt. Omoto, Ishigaki I., Ryukyus, 24. v. 1975, H. Makihara leg.; 1 ex., Yonehara, Ishigaki I., Ryukyus, 28. v. 1975, H. Makihara leg.

6. Bradymerus clathratus Schaufuss, 1887

Spec. exam.: 1 ex., Mt. Banna, Ishi., Ryukyu Is., 24. viii. 1969, H. Makihara leg. ; 10 exs., Hoshino, Ishigakijima, Nansei Is., Japan, 19. iv. 1973, Coll. H. Irie; 1 ex., Yoshihara-Arakawa, Ishigakijima, Nansei Is., Japan, 27. iv. 1974, Coll. H. Irie; Yoshihara-Arakawa, Ishigakijima, Nansei Is., Japan, 17. v. 1974, Coll. H. Irie; 1 ex., Botanical Garden, Banna, Ishigaki I., Ryukyus, 16. vi. 1974, H. Makihara leg.; 1 ex., Arakawa, Ishigaki I., Ryukyus, 18. vi. 1974, H. Makihara leg. ; 1 ex.(at light), Mt. Banna, Ishigaki I., Ryukyus, 18. vi. 1974, H. Makihara leg. 


\section{DIAPERINI}

7. Diaperis lewisi intersecta Gebien, 1913

$=$ D. sanguinipennis sinensis: M. T. Chûjô, 1975, Esakia (9) : 18 \& 19; (10) : 1-2, 5 \& 6 .

Spec, exam. : 1 ex., Mt. Banna, Ishi., Ryukyu Is., 24. viii. 1969, H. Makihara leg. ; 3 exs., Mt. Banna, Ishi., Ryukyu Is., 7-9. ix. 1969, H. Makihara leg.; 7 exs., Mt. Banna, Ishigaki, Ryukyu, 20-22. vi. 1970, H. Makihara leg. ; 2 exs., Mt. Banna, Ishigaki, Ryukyu, 30. vi. - 4. vii. 1970, H. Makihara leg. ; 3 exs. (at light), Mt. Banna, Ishigaki I., Ryukyus, 14. vi. 1974, H. Makihara leg. ; 1 ex. (at light), Botanical Garden, Banna, Ishigaki I., Ryukyus, 14. vi. 1974, H. Makihara leg. ; 1 ex.(at light), Mt. Banna, Ishigaki I., Ryukyus, 16. vi. 1974, H. Makihara leg. ; 1 ex.(at light), Mt. Banna, Ishigaki I., Ryukyus, 28. v. 1975, H. Makihara leg.; 1 ex., Mt. Banna-dake, Ishigaki I., Nansei Is., Japan, 8. vi. 1975, T. Tsutsumi leg. (Note: The elytral black bands are variable and are not always so as written in the original description.)

8. Platydema marseuli Lewis, 1894

Spec. exam.: 1 ex., Yoshiwara-Yonehara, Ishigaki I., Nansei Is., Japan, 17. vi. 1972, Coll. H. Irie; 1 ex., Tohro Riv., Ishigaki Is., 29. iii. 1973, leg. I. Matoba ; 13 exs. [host : Auricularia polytricha (Mont.) Sacc.], Mt. Omoto, Ishigaki I., Ryukyus, 9. iv. 1974, H. Makihara leg.; 1 ex., Mt. Omoto, Ishigaki I., Ryukyus, 11. iv. 1974, H. Makihara leg.; 1 ex. [host : Auricularia polytricha (Mont.) Sacc.], Mt. Omoto, Ishigaki I., Ryukyus, 12. iv. 1974, H. Makihara leg.; 1 ex., Mt. Banna, Ishigaki I., Ryukyus, 6. v. 1974, Coll. H. Irie; 1 ex., Botanical Garden, Banna, Ishigaki I., Ryukyus, 16. vi. 1974, H. Makihara leg. ; 4 exs., Arakawa, Ishigaki I., Ryukyus, 18. vi. 1974, H. Makihara leg.; 1 ex., Mt. Omoto Ishigaki I., Ryukyus, 16. iv. 1975, Coll. H. Irie.

†9. Platydema pallidicolle Lewis, 1894

Spec. exam. : 1 ex., Hoshino, Ishigakijima, Nansei Is., Japan, 19. iv. 1973, Coll. H. Irie.

\section{Platydema planicornis sp. nov.}

Oval, moderately convex. Body reddish brown, antennae gradually darkened towards apices, legs yellowish brown, elytra blackish brown, four elytral maculae dark yellow. Head sparsely punctate, clypeal suture very fine but visible, clypeus gently bent downwards at apex, front margin nearly transverse, preocular area oblique and very feebly rounded, vertex with flat and short horn, interocular area very shallowly scooped between eye and horn. Eyes strongly prominent outwards. Antennae short, scarsely reaching basal margin of pronotum, 1st antenna1 segment a little thicker than 3rd, 2nd about $2 / 5$ of 1st in length, 1st to penultimate one subequal to each other in length except for 2nd, terminal one 1.4 times as long as 1 st.

Pronotum moderately convex, nearly trapezoidal, width about twice as wide as length, very finely and sparsely punctate; subbasal foveae very shallow, front margin very narrowly marginate, front corner widely rounded, side margin very narrowly marginate, very weakly and roundly expanded, hind corner very obtuse, basal margin deeply bisinuate, not marginate. Scutellum 
semicircular, but basal margin shallowly and wholly sinuate, sparsely punctate.

Elytra finely and sparsely punctate, strongly punctate-striate, interstices very feebly convex; basal maculae slightly oblique, extending from '2nd to 8th interstices, apical maculae cover up nearly $1 / 3$ of elytra except for median line; basal margin shallowly sinuate at middle $1 / 3$, side margin narrowly marginate.

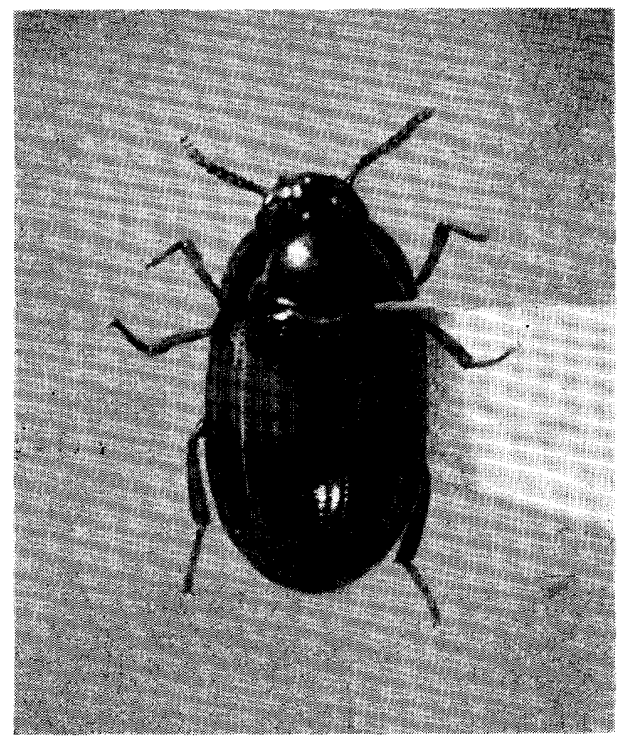

Fig. 1. Platydema planicornis sp. nov.

Gular with many transversal sutures, gular suture visible. Prosternum with golden pubescence, roughly and densely punctate ; prosternal process boat-shaped, medially grooved between front coxae. Mesonotum roughly wrinkled, V-shaped receptor of prosternal process very deep. Metasternum very sparsely pubescent, surface nearly smooth, submarginally grooved along hind coxal cavities. First to 3rd visible abdominal sternites very sparsely pubescent, roughly and evenly punctate, penultimate and terminal ones very minutely aciculate. All femora thick, comparatively flat. All tibiae feebly bent inwards, gently thickened towards apex, with many striate-setae, setae specially thick along outside. Last front tarsal segment nearly equal to 1 st to 4 th put together; hind tarsi with 1 st segment twice as long as 2nd, penultimate one shortest, terminal one a little shorter than 1st.

Length : 4.2-4.6 mm. Width : 2. 20-2.35 mm.

Distribution : Ishigakijima Island.

Type material : Holotype $\sigma^{\Uparrow}$ (Type No. 2103, Kyushu Univ.), Mt. Banna, Ishigaki Is., 22. vi. 1964, H. Konishi leg. Paratopotype : ${ }^{\star}$, same data as holotype. Paratype : ð (at light), Ishigaki City, Ryukyus, 29, v. 1975, H. Makihara leg.

Type depository: Holotype is preserved in the Entomological Laboratory, 
Faculty of Agriculture, Kyushu University.

This species is closely related to P. unicornis Gebien, 1927, from Sumatra, but is clearly distinguished from the latter by the apical maculae which cover up about the apical $1 / 3$ of the elytra.

†11. Platydema sakishimense Nakane, 1973

Spec. exam : 1 ex., Yoshiwara-Yonehara, Ishigaki I., Nansei Is., Japan, 17. vi. 1972, Coll. H. Irie; 1 ex.[host:Auricularia polytricha (Mont.) Sacc.], Mt. Omoto, Ishigaki I., Ryukyus, 9. iv. 1974, H. Makihara leg.

12. Platydema sauteri omoto Nakane, 1973

Spec. exam. : 1 ex.Lhost : Auricularia polytricha (Mont.) Sacc.], Mt. Omoto, Ishigaki I., Ryukyus, 9. iv. 1974, H. Makihara leg. ; 1 ex.(at light), Mt. Banna, Ishigaki I., Ryukyus, 9. iv. 1974, H. Makihara leg.; 1 ex., Botanical Garden, Banna, Ishigaki I., Ryukyus, 15. vi. 1974, H. Makihara leg.

\section{Platydema subfascia yaeyama Nakane, 1973}

Spec. exam. : 2 exs., Mt. Banna, Ishi., Ryukyu Is., 30. viii. 1969, H. Makihara leg.; 1 ex., Mt. Banna-dake, Is. Ishigaki, 31. viii. 1970, Coll. M. Chûjô; 3 exs., Mt. Omoto, Ishigakijima, Nansei Is., Japan, 14, iv. 1973, Coll. H. Irie; 15 exs., Mt. Banna, Ishigaki I., Ryukyus, 10. iv. 1974, H. Makihara leg.; 1 ex., Arakawa, Ishigaki I., Ryukyus, 7. vi. 1974, H. Makihara leg. ; 2 exs., Arakawa, Ishigaki I., Ryukyus, 18. vi. 1974, H. Makihara leg.; 4 exs., Botanical Garden, Banna, Ishigaki I., Ryukyus, 24. v. 1975, H. Makihara leg.

$\dagger 14$. Basanus fukudai Nakane, 1968

Spec. exam. : 6 exs., Mt. Banna, Ishi., Ryukyu Is., 24. viii. 1969, H. Makihara leg.; 1 ex., Mt. Omoto, Ishigakijima, Nansei Is., Japan, 3. iv. 1973, Coll. H. Irie; 1 ex., Mt. Omoto-dake, Ishigakijima, Nansei Is., Japan, 13. iv. 1973, Coll. H. Irie ; 1 ex., Mt. Omoto, Ishigaki I., Ryukyus, 11. iv. 1975, Coll. H. Irie.

\section{Ceropria induta induta (Wiedemann, 1819)}

Spec. exam. : 1 ex., Omoto-dake, Ishigaki, 29. x. 1963, Y. Hirashima leg.; 4 exs. (night), Mt. Banna, Ishigaki, Ryukyu, 20-22. vi. 1970, H. Makihara leg. ; 1 ex., Mt. Omoto, Ishigaki I., Ryukyus, 10. v. 1970, H. Makihara leg.; 1 ex., Mt. Banna-dake, Is. Ishigaki, 31. viii. 1970, Coll. M. Chûjô; 1 ex.(at light), Mt. Banna, Ishigaki I., Ryukyus, 12. iv. 1974, H. Makihara leg. ; 1 ex.(at light), Mt. Banna, Ishigaki I., Ryukyus, 19. vi. 1974, H. Makihara leg.

\section{LEIOCHRINI}

16. Derispia japonica Kaszab, 1961

Spec. exam. : 1 ex., Yoshihara, Ishigaki, 15. x. 1963, K. Morimoto leg.

\section{Phrenapatini}

17. Tagalus tokaranus Nakane, 1963

Spec. exam. : 1 ex., Omoto-dake, Ishigaki Is., 16. iii. 1964, C. M. Yoshimoto 
\& J. Harrell leg.

\section{ULOMINI}

†18. Tribolium (Tribolium) castaneum (Herbst, 1797)

Spec. exam. : 1 ex., Ookawa, Ishigakijima, Nansei Is., Japan, 13. iv. 1973, Coll. H. Irie.

19. Cneocnemis laminipes Gebien, 1913

Spec. exam. : 1 ex., Heigina, Ishigaki Is., 10. v. 1975, T. Takahashi leg.

20. Uloma excisa excisa Gebien, 1913

Spec. exam.: 1 ex., Omoto-dake, Ishigaki, 29. x. 1963, Y. Hirashima leg.; 1 ex., Mt. Banna, Ishi., Ryukyu Is., 24. viii. 1969, H. Makihara leg. ; 3 exs., Mt. Banna, Ishi., Ryukyu Is., 7-9. ix. 1969, H. Makihara leg. ; 2 exs., Mt. Banna, Ishigaki, Ryukyu, 20-22. vi. 1970, H. Makihara leg. ; 1 ex., Omoto, Ishigaki, 11. vii. 1970, H. Makihara leg.; 1 ex., Kabira Park, Is. Ishigaki, 23. viii. 1970, Coll. M. Chîjố; 1 ex., Mt. Omoto-dake, Ishigakijima, Nansei Is., Japan, 13. iv. 1973, Coll. H. Irie; 1 ex.(at light), Mt. Banna, Ishigaki I., Ryukyus, 12. iv. 1974, H. Makihara leg. ; 1 ex.(at light), Mt. Banna, Ishigaki I., Ryukyus, 13. iv. 1974, H. Makihara leg. ; 1 ex. (at light), Mt. Banna, Ishigaki I., Ryukyus, 25. vi. 1974, H. Makihara leg.; 1 ex., Mt. Banna, Ishigaki I., Ryukyus, 6. v. 1974, Coll. H. Irie ; 3 exs. (from decayed wood), Botanical Garden, Banna, Ishigaki I., Ryukyus, 22. x. 1974, K. Ohara leg.(emerged: 8. ii. 1975.).

$\nmid 21$. Uloma ichoi Nakane, 1956

Spec. exam. : 1 ex., Mt. Omoto, Ishigaki I., Ryukyus, 18. v. 1974, Coll. H. Irie ; 1 ex.(from decayed wood), Botanical Garden, Ishigaki I., Ryukyus, 22. x. 1974, K. Ohara leg.(emerged: 8. ii. 1975.):

$\nmid 22$. Uloma polita (Wiedemann, 1821)

Spec. exam. : 1 ex., Mt. Banna, Ishi., Ryukyu Is., 24. viii. 1969, H. Makihara leg. ; 1 ex., Mt. Banna, Ishi., Ryukyu Is., 30. viii. 1969, H. Makihara leg. ; 3 exs., Mt. Banna, Ishi., Ryukyu Is., 7-9. ix. 1969, H. Makihara leg. ; 5 exs. (night), Mt. Banna, Ishigaki, Ryukyu, 20-22. vi. 1970, H. Makihara leg. ; 5 exs., Mt. Banna, Ishigakijima, Nansei Is., Japan, 2. vi. 1972, Coll。H. Irie; 3 exs., Arakawa, Ishigaki I., Ryukyus, 7. vi. 1974, H. Makihara leg. ; 2 exs.(at light), Mt. Banna, Tshigaki I., Ryukyus, 14. vi. 1974, H. Makihara leg. ;1 ex.(at light), Mt. Banna, Ishigaki I., Ryukyus, 26. vi. 1974, H. Makihara leg. ; 2 exs. (at light), Ishigaki City, Ryukyus, 28. v. 1975, H. Makihara leg.

†23. Alphitobius diaperinus (Panzer, 1797)

Spec. exam.: 1 ex., Mt. Banna-dake, Is. Ishigaki, 31. viii. 1970, Coll. M. Chîjồ; 1 ex., Ookawa, Ishigakijima, Nansei Is., Japan, 7. v. 1974, Coll. H. Irie.

$\nmid 24$. Diaclina quadrimaculata (Gebien, 1913)

= Alphitophagus plagiatus: M. T. Chûjô, 1966, Jour. Fac. Agr., Kyushu Univ., 14(1): 14 .

Spec. exam. : 1 ex., Ishigaki Is., xi.-xii. 1952, G. E. Bohart leg. ; 10 exs., Tarogawa, Ishigaki Is., 17. iii. 1964, Y, Miyatake leg.; 4 exs., Hoshino, Ishi- 
gakijima, Nansei Is., Japan, 19. iv. 1973, Coll. H. Irie.

†25. Leptoscapha unifasciata Kaszab, 1964

Spec. exam. : 2 exs., Mt. Omoto, Ishigaki I., Ryukyus, 7. iv. 1975, Coll. H. Irie.

†26. Eutochia lateralis (Boheman, 1858)

Spec. exam. : 1 ex., Arakawa, Ishigaki I., Ryukyus, 7. vi. 1974, H. Makihara leg. ; 6 exs.(at light), Mt. Banna, Ishigaki I., Ryukyus, 14. vi. 1974, H. Makihara leg.; 1 ex.(at light), Botanical Garden, Banna, Ishigaki I., Ryukyus, 14. vi. 1974, H. Makihara leg. ; 3 exs.(at light), Mt. Banna, Ishigaki I., Ryukyus, 15. vi. 1974, H. Makihara leg.; 1 ex. (at light), Mt. Banna, Ishigaki I., Ryukyus, 16. vi. 1974, H. Makihara leg.; 1 ex., Kannondo, Ishigaki I., Ryukyus, 19. vi. 1974, H. Makihara leg.; 4 exs. (at light), Ishigaki City, Ryukyus, 23. v. 1975, H. Makihara leg.; 1 ex., Botanical Garden, Banna, Ishigaki I., Ryukyus, 24. v. 1975, H. Makihara leg.; 2 exs.(at light), Mt. Banna, Ishigaki I., Ryukyus 24. v. 1975, H. Makihara leg.; 13 exs.(at light), Ishigaki City, Ryukyus, 28. v. 1975, H. Makihara leg.

†27. Hypophloeus (Paraphloeus) amamiensis Kaszab, 1964

Spec. exam. : 1 ex., Yoshihara, Ishigaki, 15. x. 1963, K. Morimoto leg. ; 1 ex., Mt. Banna, Ishi., Ryukyu Is., 24. viii. 1969, H. Makihara leg. ; 1 ex., Mt. Banna, Ishi., Ryukyu Is., 30. viii. 1969, H. Makihara leg.

†28. Hypophloeus (Paraphloeus) gentilis (Lewis, 1894)

Spec. exam.: 7 exs. (U.S.-Jap. Co-op. Sci. Programme) (under bark), Mt. Omoto 100-526 m, Ishigaki I., Ryukyu Is., 17. xi. 1963, G. A. Samuelson leg. ;1 ex.(U. S.-Jap. Co-op. Sci. Programme) (beating), Mt. Omoto 100-526 m, Ishigaki I., Ryukyu Is., 17. xi. 1963, G. A. Samuelson leg. ; 1 ex., Mt. Banna, Ishi., Ryukyu Is., 7-9. ix. 1969, H. Makihara leg.

†29. Hypophloeus (Stenophloeus) analis Gebien, 1913

Spec. exam. : 1 ex. (U.S.-Jap. Co-op. Sci. Programme), Ishigaki I., Ryukyu Is., 21. v. 1964, T. Takara leg.

\section{TENEBRIONINI}

†30. Encyalesthus nitidipennis Fairmaire, 1898

Spec. exam.: 1 ex., Mt. Omoto-dake, Ishigakijima, Nansei Is., Japan, 13. iv. 1973, Coll. H. Irie; 3 exs., Hoshino, Ishigakijima, Nansei Is., Japan, 19. iv. 1973, Coll. H. Irie ; 3 exs. (at light), Mt. Banna, Ishigaki I., Ryukyus, 12. iv. 1974, H. Makihara leg.; 1 ex., Mt. Omoto, Ishigaki Is., 17. v. 1974, H. Hiramatsu leg. ; 1 ex.(from decayed wood), Botanical Garden, Banna, Ishigaki I., Ryukyus, 22. x. 1974, K. Ohara leg.(emerged: 8. ii. 1975.).

†31. Menephilus arciscelis Marseul, 1876

Spec. exam. : 4 exs., Mt. Banna, Ishi., Ryukyu Is., 7-9. ix. 1969, H. Makihara leg. ; 1 ex., Mt. Banna, Ishigaki, Ryukyu, 30. vi. - 4. vii. 1970, H. Makihara leg. ; 1 ex., Mt. Omoto, Ishigakijima, Nansei Is., Japan, 14. iv. 1973, Coll. H. 
Irie; 1 ex., Mt. Banna, Ishigaki I., Ryukyus, 8. v. 1974, J. Okuma leg. ; 1 ex. (at light), Mt. Banna, Ishigaki I., Ryukyus, 16. vi. 1974, H. Makihara leg.; 2 exs.(at light), Mt. Banna, Ishigaki I., Ryukyus, 25. vi. 1974, H. Makihara leg.

†32. Toxicum funginum Lewis, 1894

Spec. exam. : 1 ex.(at light), Mt. Banna, Ishigaki I., Ryukyus, 12. iv. 1974, H. Makihara leg. ; 1 ex.(at light), Mt. Banna, Ishigaki I., Ryukyus, 13. iv. 1974, H. Makihara leg. ; 1 ex., Yoshihara-Arakawa, Ishigakijima, Nansei Is., Japan, 22. v. 1974, Coll. H. Irie ; 1 ex.(at light), Arakawa, Ishigaki I., Ryukyus 11. vi. 1974, H. Makihara leg. ; 2 exs.(at light), Mt. Banna, Ishigaki I., Ryukyus, 14. vi. 1974, H. Makihara leg. ; 1 ex.(at light), Botanical Garden, Banna, Ishigaki I., Ryukyus, 14. vi. 1974, H. Makihara leg,

33. Cryphaeus satoi Kaszab, 1964

Spec. exam. : 3 exs., Arakawa, Ishigaki I., Ryukyus, 18. vi. 1974, H. Makihara leg. ; 1 ex., Mt. Omoto, Ishigaki, Nansei Is., Japan, 19. iv. 1975, Coll. H. Irie.

\section{Chodalonini}

\section{Hemicera sakishimensis sp. nov.}

Oblong-oval, comparatively strongly convex, elytra purple, with goldengreen stripes on punctate-striae, other parts shining dark brown. Head sparsely punctate, clypeal suture visible, clypeus strongly transverse, preocular area not expanded but a little convex, interocular area convex, innerocular margin grooved. Eyes strongly facetted, strongly expanded outwards.

Pronotum transverse, gently convex, sparsely punctate ; front margin widely and shallowly sinuate, narrowly marginate except for middle $1 / 10$; front corner rounded, side margin comparatively widely marginate, slightly reflexed ; hind corner obtuse; hind margin expanded basally, middle part truncate, not marginate. Scutellum very smooth, nearly triangular, each side feebly, roundly expanded. Elytra strongly convex, punctate-striate, interstices feebly convex, very sparsely aciculate ; base very slightly wider than base of pronotum, shoulder obtuse, side margin narrowly but wholly marginate, gently expanded, widest at $1 / 2$, apex rounded.

Antennae reaching middle coxae, apical 6 segments make loose club, relative length of each segment (base to apex) $=8: 4: 8: 5: 5: 6: 6: 7: 6: 6: 10$, 8th to terminal one nearly equal to each other in width, terminal one orbicular. Maxillary palpus comparatively strongly securi-formed. Gena sparsely carved. Prosternum weakly rugose ; prosternal process boat-shaped, median $1 / 3$ shallowly depressed, apex a little obtuse; propleura with several longitudinal lines; mesosternum very roughly carved, with sparse, long and yellow hairs ; protuberance wide and deep; metasternum sparsely but strongly punctate at sides. First to 3rd visible abdominal sternites with dense and longitudinal lines, median $1 / 3$ sparsely punctate; penultimate sternum very sparsely punctate, with longitudinal lines on sublateral portions; anal one very sparsely punctate. 


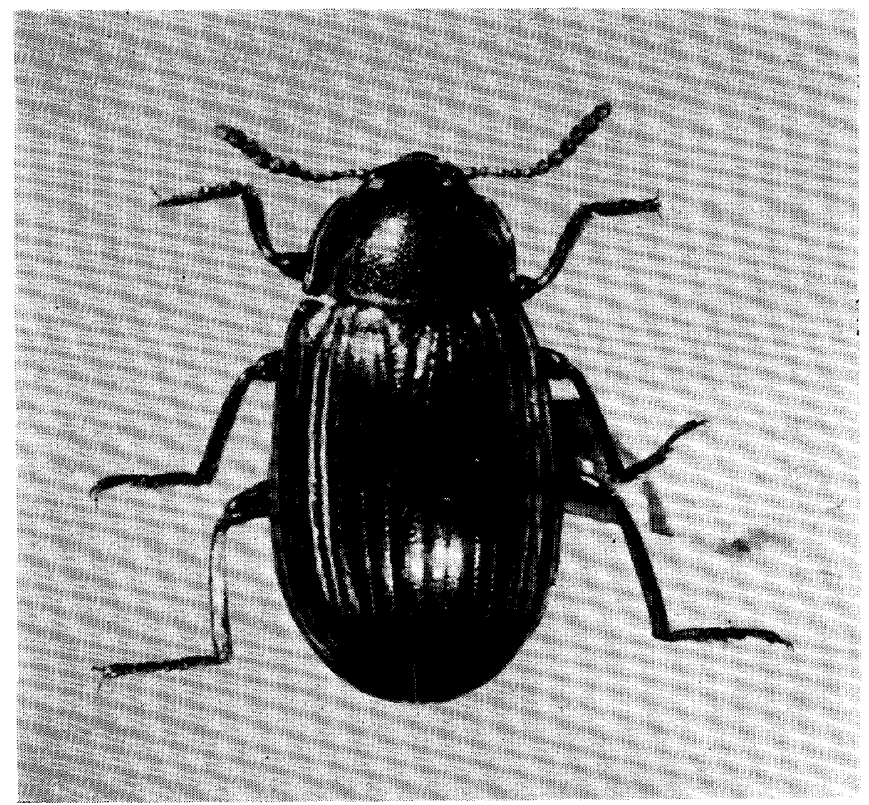

Fig. 2. Hemicera sakishimensis sp. nov.

Every femur comparatively thick, weakly flattened, sparsely aciculate, every acicula with extremely short hairs. Front tibiae with very narrow and longitudinal ridges, sparsely punctate, with yellow and brushy hairs. Front tarsi with dense, brushy and yellow hairs on sole, 1st to penultimate segments nearly equal to each other in length, terminal one a little shorter than the others put together.

Length : 4.5-8. Omm. Width: 2.5-4.2 mm.

Distribution : Sakishima Group : Miyakojima Island, Ishigakijima Island, Iriomotejima Island \& Yonakunijima Island.

Type material: Holotype $\widehat{\sigma}$ (Type No. 2104, Kyushu Univ.), Arakawa, Ishigaki I., Ryukyus, 7. vi. 1974, H. Makihara leg. Paratopotypes: $73^{7} \& 4$ 우우, same data as holotype; 3 우우, 18 . vi. 1974, H. Makihara leg. ; \& 우, 26. vi. 1974, H. Makihara leg. Paratypes: MIYAKOJIMA I. : 2 ऊठ \& 2 우우, Hirara, Miyako I., Ryukyus, 1. vi. 1975, H. Makihara leg. ISHIGAKIJIMA I.: ऊ, Mt. Omoto, Ishigaki I., Ryukyus, 9. vii. 1970, H. Makihara leg. ;우, Tohro Riv., Ishigaki Is., 29. iii. 1973, Leg. I. Matoba; 우 (at light), Mt. Banna, Ishigaki I., Ryukyus, 8. iv. 1974, H. Makihara leg. ; iv. 1974, H. Makihara leg.;2 우우, Mt. Omoto, Ishigaki I., Ryukyus, 12. iv. 1974, H. Makihara leg. ; o (at light), Mt. Banna, Ishigaki I., Ryukyus, 12. iv. 1974, H. Makihara leg. ; 2 \& $\&$, Yoshihara, Ishigaki I, Ryukyus, 28. v. 1975, H. Makihara leg. IRIOMOTEJIMA I.: $3 \gtrsim \& \&$, Otomi, Iriomote, Ryukyu Is., 12. iv. 1969, H. Makihara leg. ; 오 , Shirahama, Iri., Ryukyu Is., 31. viii. - 5. ix. 1969, H. Makihara leg. ; 우, Nakara River, Iriomote, Ryukyu, 25-28. vi. 1970, H. Makihara leg. ; o, Ohara - Mt. Goza, Iriomote I., Ryukyus, 23. vi. 1974, H. Makihara leg. ;2 3 \& 우, Kampire Fall, Iriomote, Ryukyu, 26. v. 1975, H. Makihara leg. 


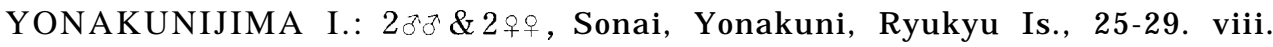
1969, H. Makihara leg. ; $3 \approx 2$ 우, Sonai -Mt. Urabu, Yonaguni I., Ryukyus, 9. vi. 1974, H. Makihara leg.

Type depository: Holotype is preserved in the Entomological Laboratory, Faculty of Agriculture, Kyushu University.

Specimens examined other than the type material: Miyakojima I., 4 \$ \& 2

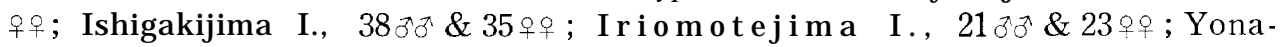
kunijima I., 26 శ̋ \& 11 우우.

This species is related to H.alternata Gebien, 1913, from Formosa, but is easily separated from the latter through the following characters:

\section{H. alternata Gebien}

Body much larger, front margin of clypeus widely sinuate, punctate-striae very fine, interstices nearly flat, visible abdominal sternites very finely and sparsely aciculate.
H. sakishimensis sp. nov.

Body much smaller, front margin of clypeus nearly straight, punctatestriae not so fine, interstices very weakly convex, visible abdominal sternites strongly but sparsely punctate.

\section{†35. Hemicera zigzaga Marseul, 1876}

Spec. exam.: 1 ex., Yoshihara, Ishigaki, 15. x. 1963, K. Morimoto leg.; 1 ex., Mt. Banna, Ishi., Ryukyu Is., 29. iv. 1969, H. Makihara leg. ; 7 exs., Mt. Banna, Ishi., Ryukyu Is., 24. viii. 1969, H. Makihara leg.; 1 ex., Mt. Banna, Ishi., Ryukyu Is., 30. viii. 1969, H. Makihara leg.; 2 exs., Mt. Banna, Ishi., Ryukyu Is., 7-9. ix. 1969, H. Makihara leg.; 1 ex., Mt. Banna, Ishigaki, Ryukyu, 2022. vi. 1970, H. Makihara leg. ; 2 exs. (night), Mt. Banna, Ishigaki, Ryukyu, 2022. vi. 1970, H. Makihara leg. ; 7 exs., Mt. Banna-dake, Is. Ishigaki, 31. viii. 1970, Coll. M. Chûjố; 1 ex., Mt. Omoto, Ishigaki I., Ryukyus, 1. vii. 1972, M. Hayashi leg. ; 3 exs., Hoshino, Ishigakijima, Nansei Is., Japan, 19. iv. 1973, Coll H. Irie ; 1 ex., Mt. Banna, Ishigaki I., Ryukyus, 28. iii. 1974, K. Kusama leg. ; 3 exs.(at light), Mt. Banna, Ishigaki I., Ryukyus, 3. iv. 1974, H. Makihara leg. ; 3 exs., Mt. Banna, Ishigaki I., Ryukyus, 9. iv. 1974, K. Kusama leg. ; 5 exs. (at light), Mt. Banna, Ishigaki I., Ryukyus, 9. iv. 1974, H. Makihara leg. ; 2 exs., Mt. Banna, Ishigaki I., Ryukyus, 10. iv. 1974, K. Kusama leg. ; 1 ex.(at light), Mt. Banna, Ishigaki I., Ryukyus, 12. iv. 1974, H. Makihara leg. ; 3 exs.(at light), Mt. Banna, Ishigaki I., Ryukyus, 13. iv. 1974, H. Makihara leg. ; 1 ex., Mt. Omoto, Ishigaki I., 1. vi. 1974, M. Sato leg.; 6 exs., Arakawa, Ishigaki I., Ryukyus, 7. vi. 1974, H. Makihara leg.; 1 ex., Botanical Garden, Banna, Ishigaki I., Ryukyus, 10. vi. 1974, H. Makihara leg.; 4 exs., Botanical Garden, Banna, Ishigaki I., Ryukyus, 12. vi. 1974, H. Makihara leg.; 2 exs.(at light), Mt. Banna, Ishigaki I., Ryukyus, 14. vi. 1974, H. Makihara leg. ; 1 ex., Botanical Garden, Banna Ishigaki I., Ryukyus, 15. vi. 1974, H. Makihara leg.; 1 ex.(at light), Mt. Banna, Ishigaki I., Ryukyus, 15. vi. 1974, H. Makihara leg.; 1 ex., Arakawa, Ishigaki I., Ryukyus, 18. vi. 1974, H. Makihara leg.; 3 exs.(at light), Mt. Banna, Ishigaki I., Ryukyus, 18. vi. 1974, H. Makihara leg. ; 2 exs.(at light), Mt. Banna, Ishigaki I., Ryukyus, 19, vi. 1974, H. Makihara leg.; 4 exs. (at light), Mt. Banna, Ishigaki I., Ryukyus, 25. vi. 1974, H. Makihara leg. ; 1 ex. (at light), Mt. Banna, Ishigaki I., Ryukyus, 26. vi. 1974, H. Makihara leg. ; 7 exs. (at light), Mt. Banna, Ishigaki I., Ryukyus, 20. v. 1975, H. Makihara leg. ; 3 exs.(at light), 
Mt. Banna, Ishigaki I., Ryukyus, 23. v. 1975, H. Makihara leg.; 2 exs.(at light), Mt. Banna, Ishigaki I., Ryukyus, 24. v. 1975, H. Makihara leg. ; 1 ex., Hirakubo, Ishigaki I., Ryukyus, 28. v. 1975, H. Makihara leg. ; 1 ex.(at light), Mt. Banna, Ishigaki I., Ryukyus, 28. v. 1975, H. Makihara leg.

†36. Plamius yaeyamensis Nakane, 1968

Spec. exam. : 1 ex., Bannadake, Ishigakijima, 1. vii. 1934, T. Esaki leg.; 1 ex., Nagura, Ishigaki I., Ryukyus, 2. ix. 1962, M. T. Chûjô leg. ; 3 exs.(malaise trap), Banna 70 m, Ishigaki I., S. Ryukyu Is., 20. v. 1964, J. L. Gressitt leg. ; 1 ex. (malaise trap), Omoto-dake $100-250 \mathrm{~m}$, Ishigaki I., S. Ryukyu Is., 22 . v. 1964, J. L. Gressitt leg.; 1 ex., Mt. Banna, Ishi., Ryukyu Is., 29. iv. 1969, H. Makihara leg.; 7 exs., Mt. Banna, Ishi., Ryukyu Is., 30. iv. 1969, H. Makihara leg. ; 8 exs.(night), Mt. Banna, Ishigaki, Ryukyu, 20-22. vi. 1970, H. Makihara leg. ; 2 exs., Mt. Omoto, Ishigaki, Ryukyu, 9. vii. 1970, H. Makihara leg. ; 2 exs., Mt. Omoto, Ishigaki, Ryukyu, 10. vii. 1970, H. Makihara leg.; 1 ex., Hoshino, Ishigakijima, Nansei Is., Japan, 19. iv. 1973, Coll. H. Irie; 3 exs., Arakawa, Ishigaki I., Ryukyus, 7. vi. 1974, H. Makihara leg.; 6 exs., Botanical Garden, Banna, Ishigaki I., Ryukyus, 10. vi. 1974, H. Makihara leg. ; 1 ex., Arakawa, Ishigaki I., Ryukyus, 11. vi. 1974, H. Makihara leg.; 1 ex., Mt. Omoto, Ishigaki I., Ryukyus, 12. vi. 1974, H. Makihara leg. ; 1 ex., Botanical Garden, Banna, Ishigaki I., Ryukyus, 14. vi. 1974, H. Makihara leg. ; 1 ex., Botanical Garden, Banna, Ishigaki I., Ryukyus, 15. vi. 1974, H. Makihara leg. ; 4 exs., Mt. Omoto, Ishigaki I., Ryukyus, 16. vi. 1974, H. Makihara leg. ; 3 exs., Arakawa, Ishigaki I., Ryukyus, 18. vi. 1974, H. Makihara leg.; 7 exs., Mt. Omoto, Ishigaki I., Ryukyus, 20. vi. 1974, H. Makihara leg.; 4 exs.(at light), Mt. Banna, Ishigaki I., Ryukyus, 25. vi. 1974, H. Makihara leg. ; 3 exs., Mt. Omoto, Ishigaki I., Ryukyus, 27. vi. 1974, H. Makihara leg. ; 2 exs., Mt. Omoto, Ishigaki I., Ryukyus, 20. v. 1975, H. Makihara leg.; 1 ex., Botanical Garden, Banna, Ishigaki I., Ryukyus, 24. v. 1975, H. Makihara leg.,. 1 ex., Yonehara, Ishigaki I., Ryukyus, 28. v. 1975, H. Makihara leg.

\section{Phaedis (Phaedis) magnipunctatus M. T. Cĥ̂jô, 1966}

Spec. exam.: 1 ex., Yoshihara, Ishigaki, 15. x. 1963, K. Morimoto leg.; 1 ex., Top of Mt. Omoto, Ishigaki I., 14. iv. 1974, H. Makihara leg. ;1 ex., Omoto Vill., Ishigaki I., 14. iv. 1974, H. Makihara leg. ; 1 ex.(at light), Mt. Banna, Ishigaki I., Ryukyus, 14. iv. 1974, H. Makihara leg. ; 6 exs., Arakawa, Ishigaki I., Ryukyus, 7. vi. 1974, H. Makihara leg.; 1 ex., Botanical Garden, Banna, Ishigaki I., Ryukyus, 12. vi. 1974, H. Makihara leg. ; 3 exs., Arakawa, Ishigaki I., Ryukyus, 18. vi. 1974, H. Makihara leg. ; 1 ex., Arakawa, Ishigaki I., Ryukyus, 26. vi. 1974, H. Makihara leg.; 2 exs., Mt. Omoto, Ishigaki I., Ryukyus, 27. vi. 1974, H. Makihara leg.

\section{Phaedis (Phaedis) marmoratus sp. nov.}

Oblong-oval, moderately convex, shining black, elytra iridescent. Head coarsely punctate, front margin of clypeus shallowly sinuate, clypeal suture deep at oblique part, preocular area not expanded, narrower than eyes, eyes transverse, innerocular groove very deep, post ocular area strongly narrowed towards neck, interocular area continuously convex to neck. Antennae short, 
not reaching basal margin of pronotum, 7th to terminal segment depressed and make loose club.

Pronotum transverse, slightly convex, sparsely but strongly punctate; front margin marginate at each $1 / 3$ of both sides, middle $1 / 3$ not marginate; front corner rounded; side margin narrowly marginate and slightly reflexed, roundly expanded, but very shallowly sinuate near hind corner as hind corner weakly prominent outwards; hind corner obtuse ; basal margin wholly marginate, faintly bisinuate. Scutellum nearly triangular, sparsely punctate. Elytra strongly convex, striate-punctures large and deep, interstices very feebly convex, sparsely aciculate ; basal margin biarcuate, faintly marginate, shoulder widely rounded, sides narrowly but wholly marginate.

Maxillary palpus strongly securi-formed. Gular to gena strongly and roughly carved. Prosternum rugose, prosternal process slightly widened at intercoxal area, from there nearly horizontal to apex, median line widely grooved, apex gently rounded. Propleura smooth at marginal half, coxal half rugose. Mesonotum strongly rugose, V-shaped receptor of prosternal process wide and comparatively shallow. Metanotum sparsely punctate, irregularly rugose. First to 3rd visible abdominal sternites very strongly and densely punctate, penultimate and terminal ones weakly and sparsely punctate.

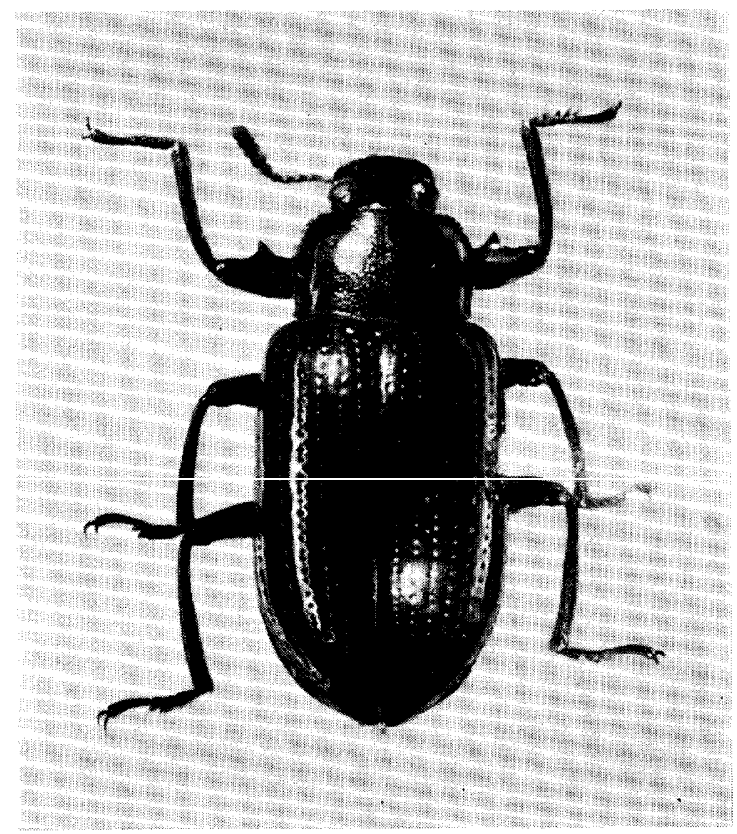

Fig. 3. Phaedis (Phaedis) marmoratus sp. nov.

Front femur thick, with thin but large dental process projecting forwards at $2 / 3$ from base. Front comparatively simple, roughly punctate, very gently thickened towards apex. Front tarsi with golden brushy hairs on sole. First hind tarsal segment nearly equal to 2 nd and penultimate one put together in length, 2nd as long as penultimate one, terminal one a little shorter than 1st 
to penultimate one put together.

Length : 10.6-12.5 mm. Width: $4.8-5.6 \mathrm{~mm}$.

Distribution: Ishigakijima Island \& Iriomotejima Island.

Type material: Holotype $\sigma^{2}$ (Type No. 2105, Kyushu Univ.), Kampire-Fall, Iriomotejima, Nansei Is., Japan, 22. iv. 1973, Coll. H. Irie. Paratopotypes: $\sigma^{\star}$ $\&$ 우 7. iv. 1973, Coll. H. Irie. Paratypes: 우, Shirahama, Iriomotejima, Nansei Is., Japan, 4. x. 1963, K. Morimoto leg. ;2 워, Shirahama, Iri., Ryukyu Is., 31. viii. - 5. ix. 1969, H. Makihara leg., 2우우, Nakara River, Iriomote, Ryukyu, 2528. vi. 1970, H. Makihara leg.; 우 , Shirahama, Iriomotejima, Nansei Is., Japan, 9, iv. 1973, Coll. H. Irie ;우, Arakawa, Ishigaki I., Ryukyus, 18. vi. 1974, H. Makihara leg. ; 오 , Mt. Omoto, Ishigaki I., Ryukyus, 20. v. 1975, H. Makihara leg. ; o , Omoto, Ishigaki Is., 2. v. 1976, T. Takahashi leg.

Type depository: Holotype is preserved in the Entomological Laboratory, Faculty of Agriculture, Kyusyu University.

This species is closely related to $P$. (P.) iridipennis Gebien, 1913, from the Philippines, but easily be distinguished from the latter through the following characters :

\section{P. iridipennis Gebien}

Body a little larger, legs bluish green, both sides of abdomen gold, pronotum nearly as broad as elytra, interstices nearly flat, tibiae nearly straight.

†39. Metaclisa nagaii Nakane, 1968

Spec, exam. : 1 ex.(at light), Mt. Banna, Ishigaki I., Ryukyus, 24. v. 1975, H. Makihara leg.; 1 ex., Mt. Omoto, Ishigaki I., Ryukyus, 29. v. 1975, H. Makihara leg.

$\uparrow 40$. Obriomaia palpalis palpaloides Nakane, 1963

Spec. exam. : 4 exs., Mt. Banna, Ishi., Ryukyu Is., 30. iv. 1969, H. Makihara leg.; 2 exs., Mt. Banna, Ishigaki, Ryukyu, 20-22. vi. 1970, H. Makihara leg. ; 2 exs., Mt. Banna, Ishigaki, Ryukyu, 30. vi. - 4. vii. 1970, H. Makihara leg. ; 1 ex., Mt. Omoto, Ishigaki, Ryukyu, 11. vii. 1970, H. Makihara leg.; 1 ex., Hoshino, Ishigakijima, Nansei Is., Japan, 19. iv. 1973, Coll. H. Irie; 1 ex., Ara. kawa, Ishigaki I., Ryukyus, 7. vi. 1974, H. Makihara leg. ; 1 ex., Botanical Graden Banna, Ishigaki I., Ryukyus, 12. vi. 1974, H. Makihara leg. ; 1 ex., Botanical Garden, Banna, Ishigaki I., Ryukyus, 14. vi. 1974, H. Makihara leg. ; 2 exs. (at light), Mt. Banna, Ishigaki I., Ryukyus, 14. vi. 1974, H. Makihara leg. ; 2 exs. (at light), Mt. Banna, Ishigaki I., Ryukyus, 16. vi. 1974, H. Makihara leg. ; 3 exs.(at light), Mt. Banna, Ishigaki I., Ryukyus, 18. vi. 1974, H. Makihara leg.; 3 exs.(at light), Mt. Banna, Ishigaki I., Ryukyus, 25. vi. 1974, H. Makihara leg. ; 1 ex.(at light), Mt. Banna, Ishigaki I., Ryukyus, 26. vi. 1974, H. Makihara leg.

†41. Obriomaia rufiventris Kaszab, 1964

Spec. exam. : 2 exs,, Mt. Banna-dake, Ishigaki I., Nansei Is., Japan, 2. vi. 1975, T. Tsutsumi leg.

42. Pseudonautes purpurivittatus (Marseul, 1876) 
Spec. exam. : 1 ex., Mt. Banna, Ishi., Ryukyu Is., 29. iv. 1969, H. Makihara leg. ; 9 exs., Mt. Banna, Ishi., Ryukyu Is., 30. iv. 1969, H. Makihara leg. ; 3 exs. Mt. Banna, Ishigaki, Ryukyu, 20-22. vi. 1970, H. Makihara leg. ; 2 exs., Mt. Omoto, Ishigaki, Ryukyu, 10. vii. 1970, H. Makihara leg. ; 1 ex., Hoshino, Ishigakijima, Nansei Is., Japan, 19. iv. 1973, Coll. H. Irie; 1 ex., Ishigaki City, Ishigaki I., Ryukyus, 14. iv. 1974, H. Makihara leg. ; 1 ex. (at light), Mt. Omoto, Ishigaki I., Ryukyus, 16. v. 1974, H. Makihara leg. ; 2 exs., Mt. Omoto, Ishigaki I., 29. v. 1974, M. Sato leg.; 5 exs., Botanical Garden, Banna, Ishigaki I., Ryukyus, 10. vi. 1974, H. Makihara leg.; 1 ex.(light trap), Mt. Banna, Ishigaki I., Ryukyus, 10. vi. 1974, H. Makihara leg. ; 1 ex.(malaise trap), Botanical Garden Banna, Ishigaki I., Ryukyus, 10-27. vi. 1974, H. Makihara leg. ; 2 exs., Arakawa, Ishigaki I., Ryukyus, 11. vi. 1974, H. Makihara leg.; 1 ex., Botanical Garden, Banna, Ishigaki I., Ryukyus, 12. vi. 1974, H. Makihara leg.; 2 exs., Botanical Garden, Banna, Ishigaki I., Ryukyus, 14. vi. 1974, H. Makihara leg.; 1 ex., Botanical Garden, Banna, Ishigaki I., Ryukyus, 15. vi. 1974, H. Makihara leg.; 2 exs.(at light), Mt. Banna, Ishigaki I., Ryukyus, 16. vi. 1974, H. Makihara leg.; 2 exs.(at light), Ishigaki I., Ryukyus, 18. vi. 1974, H. Makihara leg. ; 1 ex.(at light), Mt. Banna, Ishigaki I., Ryukyus, 19. vi. 1974, H. Makihara leg. ; 3 exs. (at light), Mt. Banna, Ishigaki I., Ryukyus, 25. vi. 1974, H. Makihara leg. ; 3 exs., Mt. Omoto, Ishigaki I., Ryukyus, 20. v. 1975, H. Makihara leg. ; 1 ex.(at light), Mt. Banna, Ishigaki I., Ryukyus, 21. v. 1975, H. Makihara leg. ; 2 exs., Mt. Omoto, Ishigski I., Ryukyus, 29. v. 1975, H. Makihara leg.; 1 ex., Botanical Garden, Ishigaki I., Ryukyus, 29. v. 1975, H. Makihara leg.

\section{Campsiomorpha imperialis Fairmaire, 1903}

Spec. exam. : 2 exs., Mt. Omoto, Is. Ishigaki, 16. vii. 1964, N. Ohbayashi leg.; 1 ex., Mt. Kabira, Ishigaki I., 29. vi. 1964, S. Ishida leg.; 3 exs., Mt. Banna, Ishi., Ryukyu Is., 24. viii. 1969, H. Makihara leg.; 15 exs., Mt. Banna, Ishigaki, Ryukyu, 20-22. vi. 1970, H. Makihara leg.; 10 exs., Mt. Banna, Ishigaki, Ryukyu, 30. vi. - 4. vii. 1970, H. Makihara leg. ; 1 ex., Mt. Omoto-dake, Is. Ishigaki, 24. viii. 1970, Coll. M. Chûjố; 10 exs.(at light), Mt. Omoto-dake, Ishigaki, 31. viii. 1970, M. Yamamoto leg. ; 1 ex., Mt. Omoto, Ishigaki I., Ryukyus, 11. vii. 1972, H. Makihara leg.; 1 ex., Botanical Garden, Banna, Ishigaki I., Ryukyus, 10. vi. 1974, H. Makihara leg.; 2 exs., Botanical Garden, Banna, Ishigaki I., Ryukyus, 15. vi. 1974, H. Makihara leg. ; 1 ex.(at light), Mt. Banna, Ishigaki I., Ryukyus, 15. vi. 1974, H. Makihara leg. ; 1 ex., Mt. Omoto, Ishigaki I., Ryukyus, 20. vi. 1974, H. Makihara leg. ; 1 ex., Mt. Omoto, Ishigaki I., Ryukyus, 25. vi. 1974, H. Makihara leg.; 1 ex., Mt. Omoto, Ishigaki I., Ryukyus, 27. vi. 1974, H. Makihara leg. ; 1 ex., Mt. Banna-dake, Ishigaki I., Nansei Is., Japan, 2. vi. 1975, T. Tsutsumi leg.

\section{HELOPIN I}

†44. Tarpela amamiensis Kaszab, 1964

Spec. exam.: 1 ex., Mt. Omoto, Ishigakijima, Nansei Is., Japan, 14. iv. 1973, Coll. H. Irie; 2 exs., Yoshihara-Yonehara, Ishigakijima, Nansei Is., Japan, 18. iv, 1973, Coll. H. Irie; 1 ex., Mt. Omoto-dake, Ishigakijima, Nansei Is., Ja- 
pan, 25. iv. 1974, Coll. H. Irie; 1 ex., Yoshihara-Arakawa, Ishigakijima, Nansei Is., Japan, 27. iv. 1974, Coll. H. Irie.

†45. Tarpela brunnea konsi Nakane, 1963

Spec. exam. : 1 ex., Mt. Omoto, Ishigakijima, Nansei Is., Japan, 3. iv. 1973. Coll. H. Irie; 1 ex., Mt. Omoto, Ishigaki I., Ryukyus, 9. iv. 1975, Coll. H. Irie.

\section{AMARYgmini}

46. Amarygmus callichromus Fairmaire, 1897

Spec. exam. : 1 ex., Tshigakijima, 23. ix. 1936, T. Masaki leg.; 1 ex., Ishigaki I., Ryukyu Is., xi.-xii. 1952, G. E. Bohart leg.; 1 ex., Hoshino, Tshigaki Is., Ryukyus, 13. iv. 1963, K. Nozato leg.; 2 exs., Taro-gawa, Ishigakijima, Ryukyus 17. iii. 1964, Y. Miyatake leg.; 1 ex., Takeda, Is. Ishigakijima, Ryukyus, 4. iv. 1968, Y. Arita leg. ; 2 exs., Mt. Banna, Ishi., Ryukyu Is., 7-9. ix. 1969, H. Makihara leg.; 4 exs., Hoshino, Ishigakijima, Nansei Is., Japan, 19. iv. 1973, Coll. H, Irie ;1 ex.(at light), Mt. Banna, Ishigaki I., Ryukyus, 13. iv. 1974, H. Makihara leg. ; 1 ex., Arakawa, Ishigaki I., Ryukyus, 7. vi. 1974, H. Makihara leg.; 1 ex., Botanical Garden, Banna, Ishigaki I., Ryukyus, 12. vi. 1974, H. Makihara leg. ; 5 exs., Arakawa, Ishigaki I., Ryukyus, 18. vi. 1974, H. Makihara leg. ;1 ex.(at light), Ishigaki City, 23. v. 1975, H. Makihara leg. ; 2 exs., Mt. Bannadake, Ishigakijima, Nansei Is., Japan, 8. vi. 1975, T. Tsutsumi leg.

47. Plesiophthalmus fuscoaenescens Fairmaire. 1898

Spec. exam. : 1 ex., Mt. Omoto, Is. Ishigaki, 31. viii. 1965, S. Ishida leg. ; 1 ex., Mt. Banna, Ishi., Ryukyu Is., 24. viii. 1969, H. Makihara leg. ; 3 exs., Mt. Banna, Ishigaki, Ryukyu, 20-22. vi. 1970, H. Makihara leg.; 1 ex., Mt. Banna, Ishigaki, Ryukyu, 30. vi. - 4. vii. 1970, H. Makihara leg. ; 2 exs., Mt. Omoto-dake, Ishigaki, 28. viii. 1970, H. Toshima leg., 1 ex., Mt. Omoto, Ishigaki I., Ryukyus, 16. v. 1974, H. Makihara leg. ; 2 exs., Arakawa, Ishigaki I., Ryukyus, 7. vi. 1974, H. Makihara leg. ; 3 exs., Botanical Garden, Banna, Ishigaki I., Ryukyus, 10. vi. 1974, H. Makihara leg.; 1 ex., Arakawa, Ishigaki I., Ryukyus, 11. vi. 1974, H. Makihara leg.; 2 exs.(at light), Botanical Garden, Banna, Ishigaki I., Ryukyus, 14. vi. 1974, H. Makihara leg., 1 ex., Mt. Omoto, Ishigaki I., Ryukyus, 20. vi. 1974, H. Makihara leg.; 1 ex., Arakawa, Ishigaki I., Ryukyus, 26. vi. 1974, H. Makihara leg.; 1 ex., Mt. Omoto, Ishigaki I., Ryukyus, 20. v. 1975, H. Makihara leg.; 1 ex., Botanical Garden, Banna, Ishigaki I., Ryukyus, 24. v. 1975, H. Makihara leg.; 2 exs., Mt. Omoto, Ishigaki I., Ryukyus, 29. v. 1975, H. Makihara leg.

†48. Elixota iridicollis Nakane, 1968

Spec. exam. : 1 ex., Mt. Banna, Ishigakijima, Nansei Is., Japan, 2. vi. 1972, Coll. H. Irie; 1 ex.(at light), Mt. Banna, Ishigaki I., Ryukyus, 24. v. 1975. H. Makihara leg.

\section{STRONGY LIINI}

\section{Strongylium cultellatum Mäklin, 1864}


Spec. exam. : 1 ex., Mt. Banna, Ishi., Ryukyu Is., 1. v. 1969, H. Makihara leg.; 1 ex., Mt. Banna, Ishigaki, Ryukyu, 30. vi. - 4. vii. 1970, H. Makihara leg. ; 1 ex., Yoshihara--Yonehara, Ishigakijima, Nansei Is., Japan, 18. iv. 1973, Coll. H. Irie; 1 ex., Yoshihara-Arakawa, Ishigakijima, Nansei Is., Japan, 27. iv. 1974, Coll. H. Irie; 2 exs., Arakawa, Ishigaki I., Ryukyus, 7. vi. 1974, H. Makihara leg. ; 3 exs., Botanical Garden, Banna, Ishigaki I., Ryukyus, 12. vi. 1974, H. Makihara leg. ; 2 exs,, Botanical Garden, Banna, Ishigaki I., Ryukyus, 14. vi. 1974, H. Makihara leg. ; 1 ex., Botanical Garden, Banna, Ishigaki I., Ryukyus, 15. vi. 1974, H. Makihara leg. ; 1 ex., Mt. Omoto, Ishigaki I., Ryukyus, 20. vi. 1974, H. Makihara leg. ; 2 exs.(at light), Mt. Banna, Ishigaki I., Ryukyus, 25. vi. 1974, H. Makihara leg.; 1 ex., Mt. Omoto, Ishigaki I., Ryukyus, 27. vi. 1974, H. Makihara leg. ; 1 ex., Botanical Garden, Banna, Ishigaki I., Ryukyus, 24. v. 1975, H. Makihara leg. ; 2 exs., Yoshihara, Ishigaki I., Ryukyus, 28. v. 1975, H. Makihara leg. ; 1 ex., Mt. Banna-dake, Ishigaki I., Nansei Is., Japan, 2. vi. 1975, T. Tsutsumi leg. ; 1 ex., Omoto, Ishigaki Is., 6. vii. 1975, T. Takahashi leg.

\section{Acknowledgements}

I wish to express my appreciation to Prof. Y. Hirashima, the head of Hikosan Biological Laboratory, for making this study possible and for his kind advice in the preparation of the manuscript. Also, I express my cordial thanks to Prof. Emeritus K. Yasumatsu, Prof. T. Shirôzu and Assoc. Prof. K. Morimoto of Kyushu University for their continual kind guidance. Particular thanks are due to Prof. Emeritus M. Chûjô of Kagawa University for his various help to my study. My sincere thanks are also due to Mr. T. Shibata of Osaka,-Mr. H. Makihara of Kyushu University and Mr. H. Irie of Fukuoka who kindly gave me so valuable Ryukyuan tenebrionid specimens. 